\title{
La chirurgia conservativa del ginocchio
}

\author{
Giovanni Pignatti \\ Reparto di chirurgia di revisione della protesi d'anca e sviluppo nuovi impianti, Istituto Ortopedico "Rizzoli", \\ Bologna, Italia \\ giovanni.pignatti@ior.it
}

(C) Società Italiana Ortopedici Traumatologi Ospedalieri d'Italia 2016

\begin{abstract}
Abbiamo voluto dedicare questo fascicolo de Lo Scalpello al trattamento conservativo della patologia degenerativa del ginocchio. L'argomento che può sembrare riduttivo, paradossalmente ci ha invece consentito di contemplare in un numero monotematico una ampia varietà di temi.

Nel fascicolo si confrontano i risultati del trattamento delle lesioni capsulo-legamentose e meniscali del ginocchio attraverso una varietà di tecniche chirurgiche; si affronta il trattamento della patologia femoro-rotulea a partire da nuovi concetti di funzionalità articolare. Inoltre si cerca di fare chiarezza su un numero sempre crescente di opzioni terapeutiche, chirurgiche e non, che indicano una generale mancanza di consenso circa il trattamento delle problematiche relative a questa complessa articolazione. Si spazia dal trattamento delle lesioni osteocondrali a quello delle deformità fino ad un più attuale inquadramento della sindrome algodistrofica con il nome "Complex Regional Pain Sindrome Tipo I".

Da questa analisi è derivato un fascicolo che abbraccia "a tutto campo" il panorama delle tecniche di chirurgia non protesica del ginocchio che può certamente fornire lo stimolo per un accrescimento culturale scientifico sia al chirurgo gio-
\end{abstract}

vane che al chirurgo maturo. Come Presidente dell'OTODI Emilia-Romagna, sono particolarmente orgoglioso di presentare questi lavori e a nome di tutto il Consiglio Direttivo Regionale rivolgo un doveroso ringraziamento al Presidente ed al CD OTODI Nazionale che ci ha concesso questa sfida; ringrazio tutti gli amici e i colleghi che con il loro contributo hanno reso possibile la realizzazione del numero monotematico.

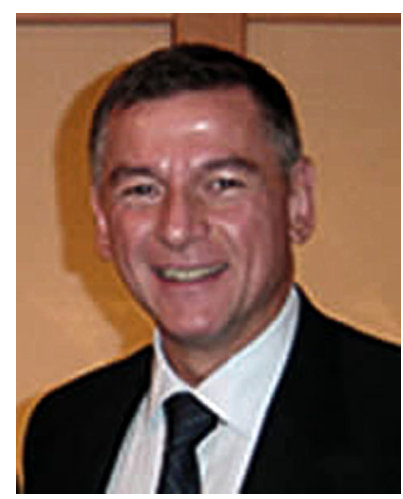

